\title{
On Self-Similarly Expanding Eshelby Inclusions: Spherical Inclusion with Dilatational Eigenstrain
}

\author{
Luqun $\mathrm{Ni}^{\text {a }}$, Xanthippi Markenscoff ${ }^{\mathrm{a}, *}$ \\ ${ }^{a}$ Department of Mechanical and Aerospace Engineering, University of California, \\ San Diego, La Jolla, California, 92093-0411
}

\begin{abstract}
For a subsonically self-similarly expanding spherical inclusion with dilatational transformation strain in a linear elastic solid, the governing system of partial differential equations is shown to be elliptic under scaling of uniform stretching of the variables, and the resulting elliptic equation is solved by satisfying the Hadamard jump conditions on the moving boundary. The solution has the Eshelby constant stress property for the interior domain, and can thus be used for the expanding inhomogeneity with transformation strain according to Eshelby (1957). The driving force on the moving boundary is also obtained.
\end{abstract}

Key words: Eshelby inclusions, transformation strain, self-similarity, elastodynamics, expanding inclusions, driving force

\footnotetext{
* Corresponding author.

Email addresses: lni@ucsd.edu (Luqun Ni), xmarkens@ucsd.edu (Xanthippi Markenscoff).
} 


\section{Introduction}

The subsonically self-similarly expanding Eshelby inclusion models for early times (Barenblatt, 1996) the motion of expanding regions of inhomogeneities with transformation strain. Expanding inclusions may be good models for deep earthquakes (Backus and Mulcahy, 1976; Rice, 1980), and also for stress waves induced martensitic transformations (Yang, Escobar and Clifton, 2009).

We consider the equation of linear elastodynamics which are invariant un-

der scaling of uniform stretch of the variables $\hat{\boldsymbol{x}}=\alpha \boldsymbol{x}, \hat{t}=\alpha t, \hat{\boldsymbol{u}}=\alpha \boldsymbol{u}$, for $\alpha>0$, with the invariance allowing for a self-similar solution in terms of the variable $\boldsymbol{u} / t$. We show that for a self-similarly expanding spherical inclusion with general eigenstrain the system of governing partial differential equations is elliptic in the domain $(|\boldsymbol{x}| / t)<\min \{a, b\}$, (with $a$ and $b$ being the pressure wave and shear speeds, respectively), while for a self-similarly expanding spherical inclusion with dilatational eigenstrain the system reduces to an elliptic ordinary differential equation, which we then solve by matching the interior Taylor expansion to the exterior Laurent expansion by the Hadamard jump conditions at the inclusion boundary. The solution exhibits the Eshelby constant stress property for the interior domain, and this can be used for solving the self-similarly expanding inhomogeneity with transformation strain by the Eshelby equivalent inclusion method.

We also obtain the driving force (per unit area) (Atkinson and Eshelby, 1968; Freund, 1972; Eshelby, 1970) on the boundary of the expanding inclusion, which is independent of time. To initiate a self-similar motion, an initial particle velocity at time $t=0$ needs to be given on the outside of the boundary of the inclusion with limiting zero radius, which is found from the solution of the problem for the jump in particle velocity across the moving boundary, which depends only on the eigenstrain and the expansion velocity $V$, independent of time. 


\section{Governing equation for a self-similarly expanding spherical in- clusion}

2.1 Governing equation for self-similarly expanding spherical inclusion with general uniform eigenstrain

A dynamic spherical inclusion with uniform transformation strain $\epsilon_{l m}^{*}$ is expanding in a self-similar manner in an infinite linear isotropic elastic medium, which is centered at the origin of a Cartesian coordinate system, and at time $t>0$, occupies the sphere

$$
\left\{\boldsymbol{x}=\left(x_{1}, x_{2}, x_{3}\right)|| \boldsymbol{x} \mid=\left(x_{1}^{2}+x_{2}^{2}+x_{3}^{2}\right)^{1 / 2} \leq V t\right\},
$$

with $V$ being the expanding speed of the spherical inclusion. The uniform eigenstrain (transformation strain) in the interior of the sphere is expressed as

$$
\epsilon_{l m}^{*}(\boldsymbol{x}, t)=\epsilon_{m l}^{*} H(V t-|\boldsymbol{x}|),
$$

where $H$ is the Heaviside step function, and $\epsilon_{m l}^{*}$ is the uniform eigenstrain. We will consider the case of dilatational eigenstrain when

$$
\epsilon_{l m}^{*}=\delta_{m l} \epsilon^{*}
$$

where $\delta$ is the Kronnecker symbol, and $\epsilon^{*}$ is the uniform dilatational eigenstrain.

The governing equations of linear elastodynamics for the displacement field $\boldsymbol{u}(\boldsymbol{x}, t)$ (Willis, 1965; Markenscoff and $\mathrm{Ni}, 2010$ ) are, for $t>0$,

$$
\rho \frac{\partial^{2} u_{j}}{\partial t^{2}}-C_{j k l m} \frac{\partial^{2} u_{l}}{\partial x_{k} \partial x_{m}}=-C_{j k l m} \epsilon_{l m}^{*} \frac{\partial}{\partial x_{k}} H(V t-|\boldsymbol{x}|)
$$


and they can be rewritten as

$$
L_{j l}\left\{\frac{\partial}{\partial t}, \nabla\right\} u_{l}=-K_{j}(\nabla) H(V t-|\boldsymbol{x}|),
$$

with the initial and boundary conditions

$$
\boldsymbol{u}\left(\boldsymbol{x}, 0^{+}\right)=\frac{\partial \boldsymbol{u}}{\partial t}\left(\boldsymbol{x}, 0^{+}\right)=0
$$

and

$$
\boldsymbol{u}(\infty, t)=0
$$

where we define

$$
\begin{aligned}
& L_{j l}\left\{\frac{\partial}{\partial t}, \nabla\right\}=\rho \frac{\partial^{2}}{\partial t^{2}} \delta_{j l}-C_{j k l m} \frac{\partial^{2}}{\partial x_{k} \partial x_{m}} \\
& K_{j}(\nabla)=C_{j k l m} \epsilon_{l m}^{*} \frac{\partial}{\partial x_{k}}
\end{aligned}
$$

with $\rho$ being the mass density of the elastic medium, $C_{j k l m}$ the elastic constants, and where and throughout, the summation convention is used.

The governing equations of linear elastodynamics (2.4) are invariant under scaling of variables $\boldsymbol{x}, t$, and the displacement $\boldsymbol{u}$. In fact, if $\boldsymbol{u}(\boldsymbol{x}, t)$ solves (2.4) and we define a scaling of uniform stretch of the variables, for $\alpha>0$,

$$
\hat{x}_{i}=\alpha x_{i}, \quad \hat{t}=\alpha t, \quad \hat{u}_{i}=\alpha u_{i},
$$

then $\hat{\boldsymbol{u}}(\hat{\boldsymbol{x}}, \hat{t})$ solves the equation

$$
\rho \frac{\partial^{2} \hat{u}_{j}}{\partial \hat{t}^{2}}-C_{j k l m} \frac{\partial^{2} \hat{u}_{l}}{\partial \hat{x}_{k} \partial \hat{x}_{m}}=-C_{j k l m} \epsilon_{l m}^{*} \frac{\partial}{\partial \hat{x}_{k}} H(V \hat{t}-|\hat{\boldsymbol{x}}|) .
$$

The initial and boundary conditions (2.6) and (2.7) are clearly invariant under the stretching of the variables. 
Markenscoff and Ni (2010) considered the expanding spherical inclusion with dilatational eigenstrain under general motion $R(t)=R_{0}+l(t)$. It can be easily shown that the motion is self-similar if and only if $R_{0}=0$ and $l(t)=V t$.

According to the general theory of dimensional analysis (Barenblatt, 1979, Bluman and Kumei, 1989), since the equation of motion (2.4) is scaling invariant, the number of its independent variables can be reduced by one, and it allows for a self-similar solution. To see this explicitly, since after the stretching of the variables, the displacement

$$
\hat{\boldsymbol{u}}(\hat{x}, \hat{t})=\alpha \boldsymbol{u}(\alpha \boldsymbol{x}, \alpha t)
$$

also solves the governing equation (2.4), from the uniqueness (Wheeler and Sternberg, 1968) of the solution of the governing equation (2.4) with the initial and boundary conditions (2.6) and (2.7), it follows that

$$
\alpha \boldsymbol{u}(\alpha \boldsymbol{x}, \alpha t)=\boldsymbol{u}(\boldsymbol{x}, t) .
$$

If we define, for $t>0$,

$$
\boldsymbol{z}=\frac{\boldsymbol{x}}{t} \quad \text { and } \quad \boldsymbol{\phi}(\boldsymbol{z}, t)=\frac{\boldsymbol{u}(\boldsymbol{x}, t)}{t}
$$

then, we have

$$
\alpha \boldsymbol{u}(\alpha \boldsymbol{x}, \alpha t)=t \phi\left(\frac{\alpha \boldsymbol{x}}{\alpha t}, \alpha t\right)=t \phi(\boldsymbol{z}, \alpha t) .
$$

From (2.13), (2.14) and (2.15), it follows that

$$
\phi(\boldsymbol{z}, \alpha t)=\boldsymbol{\phi}(\boldsymbol{z}, t)
$$

Since $\alpha>0$ is arbitrary, it follows that $\phi$ is actually independent of $t$, and

$$
\boldsymbol{u}(\boldsymbol{x}, t)=t \boldsymbol{\phi}(\boldsymbol{z})
$$


Hence, $u_{l} / t, \partial u_{l} / \partial x_{i}=\partial \phi(\boldsymbol{x} / t) / \partial\left(x_{i} / t\right)$, and components of the stress are homogeneous functions of $(\boldsymbol{x}, t)$ of order zero, and are hence self-similar.

Since Equation (2.4) is scaling invariant under scaling (2.10) of stretching coordinates, the self-similar solution of the displacement field is written in the new dependent variable $\boldsymbol{\phi}$ and new independent variable $\boldsymbol{z}=\boldsymbol{x} / t$ as

$$
u_{l}(\boldsymbol{x}, t)=t \phi_{l}\left(\frac{\boldsymbol{x}}{t}\right)=t \phi_{l}(\boldsymbol{z}) .
$$

Thus, we have

$$
\frac{\partial \boldsymbol{u}}{\partial x_{m}}=\frac{\partial}{\partial x_{m}}(t \boldsymbol{\phi}(\boldsymbol{z}))=\frac{\partial \phi}{\partial z_{m}},
$$

and

$$
\frac{\partial^{2} \boldsymbol{u}}{\partial x_{k} \partial x_{m}}=\frac{1}{t} \frac{\partial^{2} \boldsymbol{\phi}}{\partial z_{k} \partial z_{m}}
$$

Also

$$
\begin{aligned}
& \frac{\partial \boldsymbol{u}}{\partial t}=\frac{\partial}{\partial t}(t \boldsymbol{\phi}(\boldsymbol{z}))=\boldsymbol{\phi}(\boldsymbol{z})-z_{m} \frac{\partial \boldsymbol{\phi}}{\partial z_{m}}, \\
& \frac{\partial^{2} \boldsymbol{u}}{\partial t^{2}}=\frac{\partial}{\partial t}\left(\boldsymbol{\phi}(\boldsymbol{z})-z_{m} \frac{\partial \boldsymbol{\phi}}{\partial z_{m}}\right)=\frac{z_{m} z_{k}}{t} \frac{\partial^{2} \boldsymbol{\phi}}{\partial z_{k} \partial z_{m}} .
\end{aligned}
$$

By using (2.18) - (2.22), in terms of the new variables $\boldsymbol{z}$ and $\boldsymbol{\phi}$, the left hand side of Equation (2.4) is expressed as

$$
\rho \frac{\partial^{2} u_{j}}{\partial t^{2}}-C_{j k l m} \frac{\partial^{2} u_{l}}{\partial x_{k} \partial x_{m}}=\frac{1}{t}\left(\rho z_{k} z_{m} \frac{\partial^{2} \phi_{j}}{\partial z_{k} \partial z_{m}}-C_{j k l m} \frac{\partial^{2} \phi_{l}}{\partial z_{k} \partial z_{m}}\right)
$$

the right hand side of Equation (2.4) is expressed as

$$
-C_{j k l m} \epsilon_{l m}^{*} \frac{\partial}{\partial x_{k}}\left[H\left(V^{2}-|\boldsymbol{z}|^{2}\right)\right]=-\frac{1}{t} C_{j k l m} \epsilon_{l m}^{*} \frac{\partial}{\partial z_{k}}[H(V-|\boldsymbol{z}|)] .
$$

Combining (2.23) and (2.24), the governing equations of elastodynamics (2.4) for a self-similarly expanding spherical inclusion reduce to a system of partial differential equations with three independent variables $z_{i}, i=1,2,3$, 


$$
\left(\rho \delta_{j l}\left(z_{k} \frac{\partial}{\partial z_{k}}\right)\left(z_{m} \frac{\partial}{\partial z_{m}}\right)-C_{j k l m} \frac{\partial^{2}}{\partial z_{k} \partial z_{m}}\right) \phi_{l}=-C_{j k l m} \epsilon_{l m}^{*} \frac{\partial}{\partial z_{k}}[H(V-|\boldsymbol{z}|)] .
$$

By using similar definitions as (2.8) and (2.9), we have

$$
\begin{aligned}
& L_{j l}\left(z_{m} \frac{\partial}{\partial z_{m}}, \nabla_{z}\right)=\rho\left(z_{m} \frac{\partial}{\partial z_{m}}\right)^{2} \delta_{j l}-C_{j k l m} \frac{\partial^{2}}{\partial z_{k} \partial z_{m}} \\
& K_{j}\left(\nabla_{z}\right)=C_{j k l m} \epsilon_{l m}^{*} \frac{\partial}{\partial z_{k}}
\end{aligned}
$$

we rewrite Equation (2.25) as

$$
L_{j l}\left(\left(z_{k} \frac{\partial}{\partial z_{k}}\right), \nabla_{z}\right) \phi_{l}(\boldsymbol{z})=-K_{j}\left(\nabla_{z}\right) H(V-|\boldsymbol{z}|),
$$

where $\nabla_{z}=\left(\partial / \partial z_{1}, \partial / \partial z_{2}, \partial / \partial z_{3}\right)$.

Thus, by scaling of uniform stretching of the variables, we derived the governing system of partial differential equations for self-similarly expanding spherical inclusion with general eigenstrain.

\subsection{Ellipticity of the governing system of equations for self-similar expanding spherical inclusion}

We will show that the governing equation (2.25), or equivalently, (2.28), is a linear elliptic system of second order partial differential equations in the region $|\boldsymbol{z}|<\min \{a, b\}$, with $a=\sqrt{(\lambda+2 \mu) / \rho}$ and $b=\sqrt{\mu / \rho}$ being the pressure wave and shear wave speeds, where $\lambda$ and $\mu$ are Lamé's constants. It may be noted that for most elastic materials $a>b$, that is $a^{2}-b^{2}=(\lambda+\mu) / \rho=$ $(K+\mu / 3) / \rho>0$, here $K$ is the bulk modulus of the material; while there are materials having negative bulk modulus and such that $K+\mu / 3<0$, which leads to $a<b$, see e.g. Wang and Lakes (2005).

The condition for the ellipticity of a linear system of partial differential equations is discussed in, e.g., John (1955), namely, that if the determinant 
of the characteristic form of the system is nonzero, then the system is elliptic. Hence for the system (2.28), if the determinant

$$
\operatorname{det}\left[L_{j l}\left(\left(z_{k} \xi_{k}\right), \boldsymbol{\xi}\right)\right] \neq 0
$$

for all $\boldsymbol{\xi} \neq 0$, then the system (2.28) is elliptic.

It is well-known in elastodynamics that the determinant of the operator $L_{j l}$ in an isotropic medium is expressed as

$$
\begin{aligned}
& \operatorname{det}\left[L_{j l}(\gamma, \boldsymbol{\xi})\right]=\left(\rho \gamma^{2}-\mu|\boldsymbol{\xi}|^{2}\right)^{2}\left(\rho \gamma^{2}-(\lambda+2 \mu)|\boldsymbol{\xi}|^{2}\right) \\
& =\rho^{3}\left(\gamma^{2}-b^{2}|\boldsymbol{\xi}|^{2}\right)^{2}\left(\gamma^{2}-a^{2}|\boldsymbol{\xi}|^{2}\right) .
\end{aligned}
$$

Thus, for (2.26) we have

$$
\operatorname{det}\left[L_{j l}\left(\left(z_{k} \xi_{k}\right), \boldsymbol{\xi}\right)\right]=\rho^{3}\left(\left(z_{k} \xi_{k}\right)^{2}-b^{2}|\boldsymbol{\xi}|^{2}\right)^{2}\left(\left(z_{k} \xi_{k}\right)^{2}-a^{2}|\boldsymbol{\xi}|^{2}\right)
$$

If we assume $|\boldsymbol{z}|<\min \{a, b\}$, then by the Cauchy-Schwarz inequality, we have

$$
\left(z_{\kappa} \xi_{\kappa}\right)^{2} \leq|\boldsymbol{z}|^{2}|\boldsymbol{\xi}|^{2}<a^{2}|\boldsymbol{\xi}|^{2}, b^{2}|\boldsymbol{\xi}|^{2}
$$

Therefore, from (2.31) and (2.32), the condition (2.29) is satisfied, and we have proved the ellipticity of the system of partial differential equations of motion (2.28) in the region $|\boldsymbol{z}|<\min \{a, b\}$. And when $|\boldsymbol{z}|$ is outside this region, i.e., when $|\boldsymbol{z}|>\min \{a, b\}$, the system of partial differential equations (2.28) is hyperbolic.

\subsection{Governing equation for self-similarly expanding spherical inclusion with dilatational eigenstrain}

We use the spherical coordinates $r, \theta, \psi$, and the displacement vector is $\left(u_{r}, u_{\theta}, u_{\psi}\right)$. For an isotropic medium, the expanding spherical inclusion with 
dilatational eigenstrain corresponds to a spherical symmetry, which implies that the displacement components

$$
u_{\theta}=u_{\psi}=0
$$

and $u_{r}$ only depends on $r$.

Note that for an isotropic medium, we have the elastic constants

$$
C_{j k l m}=\lambda \delta_{j k} \delta_{l m}+\mu\left(\delta_{j l} \delta_{m k}+\delta_{j m} \delta_{k l}\right)
$$

Without loss of generality, we choose the field point being at $(r, 0,0)$. Then we define $z=r / t$ and $u_{r} / t=\phi(r / t)=\phi(z)$. On the right hand side of Equation (2.28), $|\boldsymbol{z}|=z$ and only the derivative with respect to $z$ is nonzero, and by using (2.3), (2.33), and (2.34), the term on the right hand side of (2.28) is reduced to

$$
(3 \lambda+2 \mu) \epsilon^{*} \frac{\partial}{\partial z} H(V-z)
$$

Moreover, by using the expression of the operator $L_{j l}$ in spherical coordinates (see, e.g., Malvern, 1969, p. 672), similarly, the left hand side of (2.28) is reduced to

$$
(\lambda+2 \mu) \frac{\partial}{\partial z}\left[z^{-2} \frac{\partial}{\partial z}\left(z^{2} \phi\right)\right]-\rho z^{2} \frac{\partial^{2}}{\partial z^{2}} \phi
$$

Therefore, the system of partial differential equations (2.28) reduces to the ordinary differential equation

$$
\left(1-\frac{z^{2}}{a^{2}}\right) \frac{d^{2} \phi}{d z^{2}}+\frac{2}{z} \frac{d \phi}{d z}-\frac{2 \phi}{z^{2}}=-\frac{(3 \lambda+2 \mu) \epsilon^{*}}{(\lambda+2 \mu)} \delta(z-V)
$$

where $\delta(\cdot)$ is the Dirac delta function. Equation (2.37) is elliptic when (1$\left.z^{2} / a^{2}\right)>0$, or $z<a$. For $z>a$, the equation of elastodynamics is hyperbolic and the uniqueness theorem requires the radiation condition at infinity, so 
that, from (2.7), with the boundary condition

$$
\phi(\infty)=0
$$

\section{Solution of the self-similarly expanding spherical inclusion with dilatational eigenstrain}

We seek the solution of Equation (2.37) with the boundary condition (2.38) for $V<a$ (subsonic motion) in the form of power series. In the interior domain of the inclusion, the solution is denoted by $\phi_{i n}$ and has a Taylor expansion, for $z<V$,

$$
\phi_{i n}(z)=\sum_{n=0}^{\infty} a_{n} z^{n}
$$

where $a_{n}$ are undetermined constants. In the exterior domain of the inclusion within $z<a$, the solution is denoted by $\phi_{\text {out }}$ and has a Laurent expansion plus an extra term to fit the delta function term on the right hand side of (2.37),

$$
\phi_{\text {out }}(z)=B(z-V) H(z-V)+\sum_{n=-\infty}^{\infty} b_{n} z^{n},
$$

where $B$ and $b_{n}$ are undetermined constants. For $z>a$, the solution is denoted by $\phi_{f a r}$, and has a Laurent expansion,

$$
\phi_{\text {far }}(z)=\sum_{n=-\infty}^{\infty} c_{n} z^{n}
$$

with $c_{n}$ being undetermined constants, which satisfies (2.37) with homogeneous right hand side since from $z>a$ and $V<a$ (subsonic motion), it follows that $\delta(z-V)=0$.

By substituting the expansions (3.1) for $\phi_{\text {in }}$ into the equation (2.37) with homogeneous right hand side

$$
\left(1-\frac{z^{2}}{a^{2}}\right) \frac{d^{2} \phi}{d z^{2}}+\frac{2}{z} \frac{d \phi}{d z}-\frac{2}{z^{2}} \phi=0,
$$


and equating the like terms, we have

$$
a_{0}=0, \quad a_{1} \neq 0, \quad a_{n}=0 \quad \text { for } \quad n \geq 2,
$$

so that

$$
\phi_{\text {in }}(z)=a_{1} z
$$

For the case $z<a$, by substituting (3.2) for $\phi_{\text {out }}$ into the equation (2.37), noting that, since $(z-V) \delta(z-V)=0$,

$$
\frac{d}{d z}[(z-V) H(z-V)]=H(z-V)
$$

and

$$
\frac{d^{2}}{d z^{2}}[(z-V) H(z-V)]=\delta(z-V)
$$

and by equating the like terms, we obtain

$$
\begin{aligned}
& B=-\left(\frac{3 \lambda+2 \mu}{\lambda+2 \mu}\right) \frac{a^{2} \epsilon^{*}}{\left(a^{2}-V^{2}\right)}, \\
& b_{0} \neq 0, \quad b_{1} \neq 0, \quad b_{-2}=-\frac{a^{2}}{3}\left(b_{0}-B V\right),
\end{aligned}
$$

while all other coefficients $b_{ \pm n}$ are zero. Thus, we have

$$
\phi_{\text {out }}(z)=B(x-V)+b_{0}+b_{1} z+\frac{b_{-2}}{z^{2}} \equiv B_{0}+B_{1} z+\frac{b_{-2}}{z^{2}},
$$

where $B_{0}=b_{0}-B V$ and $B_{1}=B+b_{1}$.

For the case when $z>a$, similarly, by substituting the expansion (3.3) into the equation (2.37) with homogeneous right hand side, and by equating the like terms, we have

$$
c_{0}+\frac{3 c_{-2}}{a^{2}}=0
$$


and all other coefficients $c_{ \pm n}$ are zero. Hence, for $z>a$, we have

$$
\phi_{f a r}(z)=c_{0}+c_{-2} z^{-2}
$$

By using the boundary condition (2.38) and Equation (3.12), from (3.13), we obtain, for $z>a$,

$$
\phi_{\text {far }}(z)=0
$$

In the solutions (3.6) and (3.11), the three undetermined constants $a_{1}, b_{0}, b_{1}$ (or equivalently, $a_{1}, B_{0}, B_{1}$ ) will be determined by three conditions: first the continuity of the displacement $u_{r}$ on the moving boundary $z=V$; second the Hadamard jump condition for the normal stress on the moving boundary $z=V$; and third the continuity of the displacement $u_{r}$ at the wave front $z=a$, as follows:

(1) On the moving boundary $r=V t$, or $z=V$, the displacement $u_{r}=t \phi(z)$ is continuous, i.e.,

$$
\phi_{\text {in }}(V)=\phi_{\text {out }}(V)
$$

which, by equating (3.6) and (3.11), gives

$$
a_{1}=\left(1-\frac{a^{2}}{3 V^{2}}\right) \frac{B_{0}}{V}+B_{1} .
$$

(2) on the moving boundary $r=V t$, or $z=V$, the Hadamard jump condition

$$
[[\boldsymbol{\sigma} \cdot \boldsymbol{n}]]=\left[\left[\sigma_{r r}\right]\right]=-\rho V\left[\left[\frac{\partial u_{r}}{\partial t}\right]\right]
$$

is satisfied where the symbol [[.]] denotes the jump of a variable across the interface, and is defined by

$$
[[A]]=A^{+}-A^{-}
$$

with $A^{+}$and $A^{-}$being the limiting values of $A$ from either side of the interface, respectively, provided that the limits exist. 
Notice that at the field point $(r, 0,0)$,

$$
\sigma_{r r}=(\lambda+2 \mu) \frac{\partial u_{r}}{\partial r}+2 \lambda \frac{u_{r}}{r}-C_{11 j k} \epsilon_{j k}^{*}
$$

So that on the moving boundary $r=V t$, we have

$$
\left[\left[\sigma_{r r}\right]\right]=(\lambda+2 \mu)\left[\left[\frac{\partial u_{r}}{\partial r}\right]\right]+(3 \lambda+2 \mu) \epsilon^{*}
$$

since $\left[\left[u_{r}\right]\right]=0$. We compute the jumps $\left[\left[\partial u_{r} / \partial t\right]\right]$ and $\left[\left[\partial u_{r} / \partial r\right]\right]$ in (3.17) and (3.20). From (3.6) and (3.11), we have

$$
\left(u_{r}\right)_{\text {in }}=t \phi_{\text {in }}=a_{1} r
$$

and

$$
\left(u_{r}\right)_{\text {out }}=t \phi_{\text {out }}=t\left(1-\frac{a^{2} t^{2}}{3 r^{2}}\right) B_{0}+B_{1} r .
$$

From (3.21) and (3.22), we have

$$
\frac{\partial\left(u_{r}\right)_{\text {in }}}{\partial t}=0, \quad \frac{\partial\left(u_{r}\right)_{\text {out }}}{\partial t}=\left(1-\frac{a^{2} t^{2}}{r^{2}}\right) B_{0}
$$

and

$$
\frac{\partial\left(u_{r}\right)_{\text {in }}}{\partial r}=a_{1}, \quad \frac{\partial\left(u_{r}\right)_{\text {out }}}{\partial r}=\frac{2 a^{2} t^{3}}{3 r^{3}} B_{0}+B_{1} .
$$

From (3.23) and (3.24), we have the jumps at the moving boundary $r=$ $V t$ :

$$
\begin{aligned}
& {\left[\left[\frac{\partial\left(u_{r}\right)}{\partial t}\right]\right]=-\frac{\left(a^{2}-V^{2}\right)}{V^{2}} B_{0},} \\
& {\left[\left[\frac{\partial\left(u_{r}\right)}{\partial r}\right]\right]=\frac{\left(a^{2}-V^{2}\right)}{V^{3}} B_{0} .}
\end{aligned}
$$

From (3.20) and (3.26), it follows

$$
\left[\left[\sigma_{r r}\right]\right]=(\lambda+2 \mu) \frac{\left(a^{2}-V^{2}\right)}{V^{3}} B_{0}+(3 \lambda+2 \mu) \epsilon^{*} .
$$

Then substituting (3.16), (3.25) and (3.27) into the Hadamard jump condition (3.17), we obtain the value $B_{0}$ :

$$
B_{0}=-\frac{(3 \lambda+2 \mu) a^{2} V^{3} \epsilon^{*}}{(\lambda+2 \mu)\left(a^{2}-V^{2}\right)^{2}} .
$$


(3) By the continuity of the displacement $u_{r}$ at the wave front $r=a t$, or $z=$ $a$, from (3.14), $\phi_{f a r}=0$, hence, we have that the limit of the displacement from $r<$ at to $r=a t$ is zero,

$$
\lim _{r \rightarrow(a t)^{-}} u_{r}(r)=0
$$

Thus, from the expression (3.22), we have

$$
\left(u_{r}\right)_{\text {out }}(a t)=t\left(\frac{2}{3} B_{0}+B_{1} a\right)=0
$$

which leads to

$$
B_{1}=-\frac{2}{3 a} B_{0}
$$

Thus, from (3.16), (3.28), and (3.31), we have

$$
\begin{aligned}
& a_{1}=\frac{(3 \lambda+2 \mu)}{(\lambda+2 \mu)} \frac{a(2 V+a) \epsilon^{*}}{2(a+V)^{2}}, \\
& B_{0}=-\frac{(3 \lambda+2 \mu)}{(\lambda+2 \mu)} \frac{a^{2} V^{3} \epsilon^{*}}{\left(a^{2}-V^{2}\right)^{2}}, \\
& B_{1}=\frac{2(3 \lambda+2 \mu)}{3(\lambda+2 \mu)} \frac{a V^{3} \epsilon^{*}}{\left(a^{2}-V^{2}\right)^{2}} .
\end{aligned}
$$

Therefore, we have the following solution for the field quantities:

(1) The displacement

For a point in the interior domain of the inclusion $r<V t$, or, $z<V$

$$
u_{r} \text { (interior) }=\frac{(3 \lambda+2 \mu) \epsilon^{*} r(2 V+a)}{3 \rho a(V+a)^{2}},
$$

for a point in the exterior domain of the inclusion $V t<r<a t$, or, $V<z<a$,

$$
u_{r}(\text { exterior })=\frac{(3 \lambda+2 \mu) \epsilon^{*} V^{3}(a t-r)^{2}(2 r+a t)}{3 \rho a\left(a^{2}-V^{2}\right)^{2} r^{2}} .
$$

(2) The displacement gradient

For $r<V t$,

$$
\frac{\partial u_{r}}{\partial r} \text { (interior) }=\frac{(3 \lambda+2 \mu) \epsilon^{*}(a+2 V)}{3 \rho a(a+V)^{2}},
$$


and, for $a t>r>V t$,

$$
\frac{\partial u_{r}}{\partial r}(\text { exterior })=\frac{2(3 \lambda+2 \mu) \epsilon^{*} V^{3}\left(r^{3}-a^{3} t^{3}\right)}{3 \rho a\left(a^{2}-V^{2}\right)^{2} r^{3}} .
$$

(3) The particle velocity

For $r<V t$,

$$
\frac{\partial u_{r}}{\partial t}(\text { interior })=0,
$$

and, for $a t>r>V t$,

$$
\frac{\partial u_{r}}{\partial t}(\text { exterior })=\frac{(3 \lambda+2 \mu) \epsilon^{*} V^{3}\left(a^{2} t^{2}-r^{2}\right)}{\rho\left(a^{2}-V^{2}\right)^{2} r^{2}} .
$$

(4) The particle acceleration

For $r<V t$,

$$
\frac{\partial^{2} u_{r}}{\partial t^{2}} \text { (interior) }=0,
$$

and, for $a t>r>V t$,

$$
\frac{\partial^{2} u_{r}}{\partial t^{2}} \text { (exterior) }=\frac{2(3 \lambda+2 \mu) \epsilon^{*} V^{3} a^{2} t}{\rho\left(a^{2}-V^{2}\right)^{2} r^{2}} .
$$

For $r>a t$, all field quantities are zero. The above solutions are consistent with the results in Xiao and Luo (2003), and Markenscoff and Ni (2010).

Equation (3.37) shows that the displacement gradient in the interior domain of the inclusion is constant and independent of the position, thus in the interior domain of the inclusion the stress, which is given by

$$
\sigma_{m l}=C_{m l j k}\left(\frac{\partial u_{j}}{\partial x_{k}}-\epsilon_{j k}^{*}\right),
$$

is also constant, that is the constant stress Eshelby property in the interior domain.

Equations (3.25) and (3.28) give the jump of the particle velocity across the moving inclusion boundary $r=V t$ :

$$
\left[\left[\frac{\partial u_{r}}{\partial t}\right]\right]=\frac{(3 \lambda+2 \mu) \epsilon^{*} V}{\rho\left(a^{2}-V^{2}\right)} .
$$


We note that this is independent of time and is also the value of the particle velocity on the outside of the inclusion in the limiting time $t \rightarrow 0$ when the radius of the inclusion $r=V t$ also tends to zero. Thus Equation (3.44) gives the initial condition of the particle velocity (on the outside surface of the inclusion) needed to initiate the self-similar expanding motion with velocity $V$ for a spherical inclusion with dilatational eigenstrain $\epsilon^{*}$. All other points have initial particle velocity zero and the initial displacement is also zero.

\section{The driving force on the moving boundary of a self-similarly expanding spherical inclusion with dilatational eigenstrain}

The driving force (configurational force, or self force) on the boundary $|\boldsymbol{x}|=V t$ of the self-similarly expanding spherical inclusion with dilatational eigenstrain is defined in terms of the energy-release rate for a moving singularity. The energy-release rate for a moving defect with velocity $\boldsymbol{v}$ was defined by Eshelby (1970), Freund (1972, 1990), and Markenscoff and Singh (2015), as the net flux through a contour $S$ surrounding the defect, moving with it and shrinking onto it:

$$
\dot{\mathcal{E}}=-\lim _{S \rightarrow 0} \int_{S}\left[n_{j} \sigma_{i j} \dot{u}_{j}+v_{n}(W+T)\right] d S,
$$

where $\boldsymbol{n}=\left(n_{1}, n_{2}, n_{3}\right)$ is the outward normal of the moving boundary, $v_{n}=(\boldsymbol{v}$. $\boldsymbol{n}), W$ and $T$ are the potential and kinetic energy densities, respectively. It was proved by (Freund and Clifton, 1974) that, for any defect less singular than the crack $\left(r^{-1 / 2}\right.$ singular), the energy-release rate (4.1) is path-independent. Thus it is also path-independent when the defect is a jump in the field quantities as for the moving boundary of the self-similar expanding spherical inclusion.

We choose the integral surface $S_{\epsilon}$ as two spheres $|\boldsymbol{x}|=V t \pm \epsilon$ enclosing the moving boundary $S_{d}$, the sphere $|\boldsymbol{x}|=V t$, of the expanding spherical inclusion for an infinitesimal number $\epsilon$. The energy-release rate is 


$$
\begin{aligned}
\dot{\mathcal{E}} & =-\lim _{S_{\epsilon} \rightarrow S_{d}} \int_{S_{\epsilon}}\left[n_{j} \sigma_{i j} \dot{u}_{j}+v_{n}(W+T)\right] d S \\
& =\int_{S_{d}}\left(n_{j}\left[\left[\sigma_{i j} \dot{u}_{j}\right]\right]+V[[W+T]]\right) d S .
\end{aligned}
$$

Note the identity

$$
[[A B]]=<A>[[B]]+[[A]]<B>
$$

where we define the symbol $<>$ by

$$
<A>=\frac{1}{2}\left(A^{+}+A^{-}\right) .
$$

Using (4.3), we have

$$
\begin{array}{r}
n_{j}\left[\left[\sigma_{i j} \dot{u}_{j}\right]\right]=n_{j}<\sigma_{i j}>\left[\left[\dot{u}_{i}\right]\right]+n_{j}\left[\left[\sigma_{i j}\right]\right]<\dot{u}_{i}> \\
=-V n_{j}<\sigma_{i j}>\frac{\partial u_{i}}{\partial \boldsymbol{n}}-\frac{V}{\rho}\left[\left[\dot{u}_{i}\right]\right]<\dot{u}_{i}> \\
=-V<\sigma_{i j}>\frac{\partial u_{i}}{\partial x_{j}}-\frac{V}{\rho}\left[\left[\dot{u}_{i}\right]\right]<\dot{u}_{i}>.
\end{array}
$$

where the following well-known Hadamard jump conditions across the interface are used

$$
\begin{aligned}
n_{j}\left[\left[\sigma_{i j}\right]\right] & =-V \rho\left[\left[\dot{u}_{i}\right]\right], \\
{\left[\left[\dot{u}_{i}\right]\right] } & =-V\left[\left[\frac{\partial u_{i}}{\partial \boldsymbol{n}}\right]\right],
\end{aligned}
$$

and the following relation (Eshelby,1970) is used

$$
\frac{\partial u_{i}}{\partial \boldsymbol{n}} n_{j}=\frac{\partial u_{i}}{\partial x_{j}}
$$

And notice that

$$
[[T]]=\frac{1}{\rho}<\dot{u}_{i}>\left[\left[\dot{u}_{i}\right]\right] .
$$


Substituting (4.5) and (4.9) into (4.10), we have

$$
\dot{\mathcal{E}}=\int_{S_{d}}-V\left([[W]]-<\sigma_{i j}>\left[\left[\frac{\partial u_{i}}{\partial x_{j}}\right]\right]\right) d S .
$$

The driving force per unit area in the direction of the normal to the boundary is written as

$$
f=[[W]]-<\sigma_{i j}>\left[\left[\frac{\partial u_{i}}{\partial x_{j}}\right]\right] .
$$

For inclusions, the strain and stress are given as

$$
\epsilon_{i j}=\frac{\partial u_{i}}{\partial x_{j}}-\epsilon_{i j}^{*}, \quad \sigma_{i j}=C_{i j k m}\left(\frac{\partial u_{k}}{\partial x_{m}}-\epsilon_{k m}^{*}\right),
$$

we have

$$
[[W]]=\frac{1}{2} C_{i j k m}\left[\left[\left(\frac{\partial u_{k}}{\partial x_{m}}-\epsilon_{k m}^{*}\right)\left(\frac{\partial u_{i}}{\partial x_{j}}-\epsilon_{i j}^{*}\right)\right]\right]=<\sigma_{i j}>\left[\left[\frac{\partial u_{i}}{\partial x_{j}}-\epsilon_{i j}^{*}\right]\right] .
$$

From (4.11) and (4.13), the driving force on the moving boundary of expanding inclusion is given by (Markenscoff and $\mathrm{Ni}, 2010$ )

$$
f=-<\sigma_{m l}>\left[\left[\epsilon_{m l}^{*}\right]\right]=<\sigma_{m l}>\epsilon_{m l}^{*}
$$

Using (4.12) and the dilatational eigenstrain (2.3) in (4.14), we have

$$
\begin{aligned}
f & =<\tau_{11}+\tau_{22}+\tau_{33}>\epsilon^{*}-\frac{3}{2}(3 \lambda+2 \mu) \epsilon^{* 2} \\
& =<\tau_{r r}+\tau_{\theta \theta}+\tau_{\phi \phi}>\epsilon^{*}-\frac{3}{2}(3 \lambda+2 \mu) \epsilon^{* 2},
\end{aligned}
$$

where

$$
\tau_{m l}=C_{m l j k} \frac{\partial u_{j}}{\partial x_{k}}
$$

are the components of the elastic stress tensor in Cartesian coordinates, and $\tau_{r r}, \tau_{\theta \theta}, \tau_{\phi \phi}$ are the components in spherical coordinates, which are expressed 


$$
\begin{aligned}
\tau_{r r} & =(\lambda+2 \mu) \frac{\partial u_{r}}{\partial r}+2 \lambda \frac{u_{r}}{r}, \\
\tau_{\theta \theta} & =\tau_{\phi \phi}=\lambda \frac{\partial u_{r}}{\partial r}+2(\lambda+\mu) \frac{u_{r}}{r} .
\end{aligned}
$$

Hence by substituting (4.17) and (4.18) into (4.15) and using the solution for $u_{r}$ inside and outside the inclusion given in (3.35) and (3.36), we obtain the driving force on the moving boundary $r=V t$, as

$$
\begin{aligned}
f & =(3 \lambda+2 \mu)<\frac{\partial u_{r}}{\partial r}+2 \frac{u_{r}}{r}>\epsilon^{*}-\frac{3}{2}(3 \lambda+2 \mu) \epsilon^{* 2} \\
& =-\frac{2 \mu(3 \lambda+2 \mu) \epsilon^{* 2}}{(\lambda+2 \mu)}-\frac{(3 \lambda+2 \mu)^{2} \epsilon^{* 2} V^{2}(3 a-V)}{2(\lambda+2 \mu)(a+V)\left(a^{2}-V^{2}\right)}
\end{aligned}
$$

We notice that the driving force is independent of time. The first term in (4.19) coincides with the driving force for the static inclusion as given by Eshelby (1970) and Gavazza (1977).

\section{Conclusions}

The early motion of a subsonically expanding Eshelby spherical inclusion with transformation strain is captured by the self-similar motion which is obtained here within the framework of the self-similar equations of elastodynamics which become elliptic. The solution was obtained and it exhibits the constant stress Eshelby property in the interior domain, which allows for the application of the equivalent inclusion method for expanding inhomogeneities. The jump condition for the particle velocity obtained by the solution determines the initial condition for a particular expansion velocity. The driving force on the inclusion boundary is also computed.

\section{Acknowledgement}

This work was supported by National Science Foundation grant CMS 1129888. 


\section{References}

Atkinson, C. and Eshelby, J.D. (1968) The flow of energy into the tip of a moving crack. Int. J. Fracture Mechanics, 4, 3-8.

Backus, G. and Mulcahy, M. (1976) Moment tensors and other phenomenological descriptions of seismic sources-I. Continuous displacement. Geophys. J.R. astr. Soc., 46, 341-361.

Barenblatt, G.I. (1996) Scaling, Self-Similarity, and Intermediate Asymptotics, Cambridge University Press.

Bluman, G.W. and Kumei, S. (1989) Symmetries and Differential Equations, Spring-Verlag.

Eshelby, J.D. (1970) Energy relation of the energy-momentum tensor in continuum mechanics. In Inelastic Behavior of Solids, Eds. M.F. Kannien, W.F. Adler, A.R. Rosenfeld, and R.I. Taffee, 77-115.

Eshelby, J.D. (1977) Lecture on the elastic energy-momentum tensor. In Collected Works of J.D. Eshelby: The Mechanics of Defects and Inhomogeneities, Eds. Markenscoff, X. and Gupta, A., Springer, Netherlands, 2006, 907-931.

Freund, L.B. (1972) Energy flux into the tip of an extending crack in an elastic solid. J. Elasticity, 2, 341-349.

Freund, L.B. and Clifton, R.J. (1974) On the uniqueness of elastodynamic solution for running cracks. J. Elasticity, 4, 293-299.

Freund, L.B. (1990) Dynamic Fracture Mechanics, Cambridge University Press, Cambridge.

John, F. (1955) Plane Waves and Spherical Means Applied to partial differential Equations, Interscience; New York.

Gavazza, S.D. (1977) Forces on pure inclusion and Somigliana dislocations. Scr. Metal. 11, 979-981.

Malvern, L.E. (1969) Introduction to The Mechanics of a Continuous Medium, Prentice-Hall.

Markenscoff, X. and Ni, L. (2010) The energy-release rate and self-force of dynamically expanding spherical and plane inclusion boundaries with di- 
latational eigenstrain. J. Mech. Phys. Solids, 58, $1 \mathrm{C} 11$.

Markenscoff, X. and Singh, S.P.V. (2015) Dynamic conservation integrals as dissipative mechanisms in the evolution of inhomogeneities. Journal of Mechanics of Materials and Structures, to appear.

Rice, J.R. (1980) The mechanics of earthquake rupture. In Physics of the Earth's Interior, Proc. Int. School of Physics "E. Fermi", Course 78, 1979, Eds. A.M. Dziewonski and E. Boschi, Italian Physical Society North Holland Publ. Co.

Wang, Y.C. and Lakes, R.S. (2005) Composites with inclusions of negative bulk modulus: extreme damping and negative Poisson's ratio. Journal of Composits Materials, 39, No. 18, 1745-1758.

Wheeler, L.T. and Sternberg, E. (1968) Some Theorems in classical elastodynamics. Arch. Rational Mech Anal., 31, 51-90.

Willis, J.R. (1965) Dislocations and inclusions. J. Mech. Phys. Solids, 13, 337-395.

Xiao, Z.M. and Luo, J. On the transient elastic field for an expanding spherical inclusion in 3-D solid. Acta Mech., 163, 147-159.

Yang, S.Y., Escobar, J. and Clifton, R.J. (2009) Computational modeling of stress-wave-induced martensitic phase transformations in NiTi. Mathematics and Mechanics of Solids, 14, 220-257. 